\title{
Parametric Design Used in the Creation of 3D Models with Weaving Characteristics
}

\author{
Hui-Chin Chang \\ Department of Creative Product Design, HungKuo Delin University of Technology, Taiwan \\ Email: chang.hcjang@gmail.com
}

How to cite this paper: Chang, H.-C. (2021) Parametric Design Used in the Creation of 3D Models with Weaving Characteristics. Journal of Computer and Communications, 9, 112-127.

https://doi.org/10.4236/jcc.2021.911008

Received: October 23, 2021

Accepted: November 20, 2021

Published: November 23, 2021

Copyright $\odot 2021$ by author(s) and Scientific Research Publishing Inc. This work is licensed under the Creative Commons Attribution International License (CC BY 4.0).

http://creativecommons.org/licenses/by/4.0/ (c) (i) Open Access

\begin{abstract}
The art of weaving is an ancient and beautiful technique that never fades. Various weaving techniques and various totem patterns contain rich cultural connotations. The development of cultural and creative industries often uses existing environmental materials to deconstruct and use them in innovative ways to reinspire and present another style. With the rapid progress of computer-aided design technology, digital applications in the design practice has become an important element, and parametric design is the best popular design method in recent years. This paper is mainly in view of the weaving crafts in the traditional culture of the world occupies an important role, and the precious traditional weaving crafts gradually lost. Therefore, it is planned to construct a 3D model with knitting characteristics through a parametric design method, and print out the prototype through the $3 \mathrm{D}$ printing mechanism, that is, the traditional craftsmanship is integrated into the modern manufacturing process with innovative techniques, to show the new style of this weaving craft culture that is different from the past, so that it not only retain its inherent spirit, but also can promote this ideal of cultural creativity.
\end{abstract}

\section{Keywords}

Weaving Crafts, Parametric Design, Grasshopper, 3D Printer

\section{Preface}

The art of weaving is an ancient and beautiful technique that never fades. Various weaving techniques and various totem patterns contain rich cultural connotations. The development of cultural and creative industries often uses existing environmental materials to deconstruct and use them in innovative ways to reinspire and present another style, continue the meaning of existing cultural materials, and create infinity for a new culture. However, this precious traditional 
craft of weaving is gradually being lost. Therefore, how to integrate traditional crafts into modern manufacturing processes with innovative techniques to continue and preserve this traditional crafts should be the place where those who are currently engaged in the cultural and creative industries need to work hard [1].

The early weaving crafts were closely integrated with people's lives, and gradually elevated to the artistic level, turning them into handicrafts for appreciation. Moreover, due to the rise of cultural and creative concepts, consumers' requirements for product quality have begun to change, and hand-made products with exquisite designs have gradually been welcomed by the market. And the cultural and creative related industries with the concept of cultural thinking, driven by the government's efforts, more people have invested in trying to bring art and beauty into daily necessities, so it is not difficult to find its delicate weaving on objects in daily life [2].

With the rapid advancement of Computer-Aided Design related technologies, digital applications have become an indispensable part of design practice. Parametric design has become a popular new design method in this design field in recent years. Different from the 3D modeling software commonly used in product design, the parametric design tool is a design method in which parameters and calculation logic are connected to each other to generate geometric patterns. The changes in the geometric model can be seen in real time through the adjustment of parameters and calculation elements. At the same time, the breakthroughs in digital manufacturing technology in recent years have given designers more new possibilities in design [3].

Like its name, parametric design presents all elements of the design process as data. The geometric shape is no longer determined by the graphics drawn by paper and pen, but by the direct construction of data and shape logic. Its design and construction method is not as in the modeling software that we are familiar with, in the 3D working space with elements such as points, lines and planes, intuitively performing shapes, stacking, stretching and other actions to complete the design methods of geometric modeling. The design is woven with the arrangement and interactive links between data on a blank paper-like operating platform [4].

The application of data gives the definitions and rules behind the natural patterns a suitable stage. The concept of parametric design allows mathematical definitions and even various algorithms to be added to design ideas. Through the combination of data and algorithms, the shapes can be creating based on specific logic or mathematical definitions. In the development of digital architecture, there have been many practical cases of algorithms that use natural patterns for design creation. With parametric design techniques, people's ideas of "real" natural designs seem to be realized [5].

The development of architectural design and industrial design is inseparable. Although parametric design has been widely used in the field of architectural design, the information on industrial design is still extremely limited. Although some works of parametric designs have appeared one after another, but the dis- 
cussion on the application of the entire design process is still at a relatively inadequate stage, especially in the cultural and creative industry, and no one mentions its application [6] [7] [8].

In addition to analyzing the structure of weaving features, this paper also introduces the method of traditional modeling techniques to construct the weaving features. At the same time, it gradually analyzes the entire process of constructing a 3D model with weaving features using GH parametric design. Finally, the prototype is made through the $3 \mathrm{D}$ printing mechanism, that is, the traditional craftsmanship is integrated into the modern manufacturing process with innovative techniques, to show the new style of this weaving craft culture that is different from the past, so that it not only retain its inherent spirit, but also can promote this ideal of cultural creativity.

\section{Weaving Product Structure Analysis}

From its simplest structure, the weaving process can be regarded as two sets of parallel yarns, and the two sets of parallel yarns are mutually orthogonal and interlaced. If you observe its structure more closely, you can see that the two sets of parallel yarns undulate up and down in a wavy shape, passing through each other, never intersecting. This structure still needs to be determined according to the smoothness requirements of the textile. On fabrics that need to be smoother, the weft yarn is usually straight, while the warp yarn undulates up and down in a wavy shape, and passes through the weft yarn, as shown in Figure 1.

On the other hand, if the fabric needs to be rougher or more complicated, the warp and weft will undulate up and down in a wavy shape, and pass through each other, as shown in Figure 2.

\section{The Traditional Construction Process of Products with Weaving Characteristics}

As mentioned earlier, the weaving product structure can be regarded as two sets of parallel yarns that are orthogonal to each other and interlaced in a wave-like up and down, crossing each other, never intersecting. Therefore, the steps for constructing the weaving feature should be as follows:

Step 1: Determine the surface to be constructed, as shown in Figure 3.

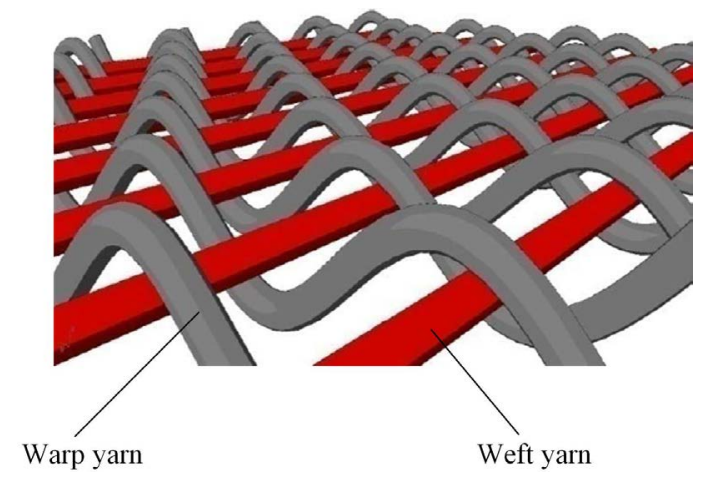

Figure 1. Smooth fabric structure [9]. 
Step 2: Determine the number of warp and weft to get the number of dividing points, as shown in Figure 4.

Step 3: Determine the wave height.

Step 4: According to the wave height and the number of wefts, two data groups are generated: "Weft positive dividing points group" and "Weft negative dividing points group", as shown in Figure 5.

Step 5: Use the B-Spline curve to connect the dividing points of each weft direction to generate multiple wave trajectories in series, as shown in Figure 6.

Step 6: Copy the original surface as the basis for generating the dividing points in the warp direction.

Step 7: Swap the UV direction of the copied surface to control the weft direction and the warp direction to be perpendicular to each other.

Step 8: Repeat Step 4 to Step 5 to complete the wave trajectories in the warp direction.

Step 9: Change all wave trajectories to round-head pipes with appropriate diameters to complete the finished pipe weaving characteristic, as shown in Figure 7.

And Figure 8 is the flow chart of traditional construction process of products with weaving characteristics.

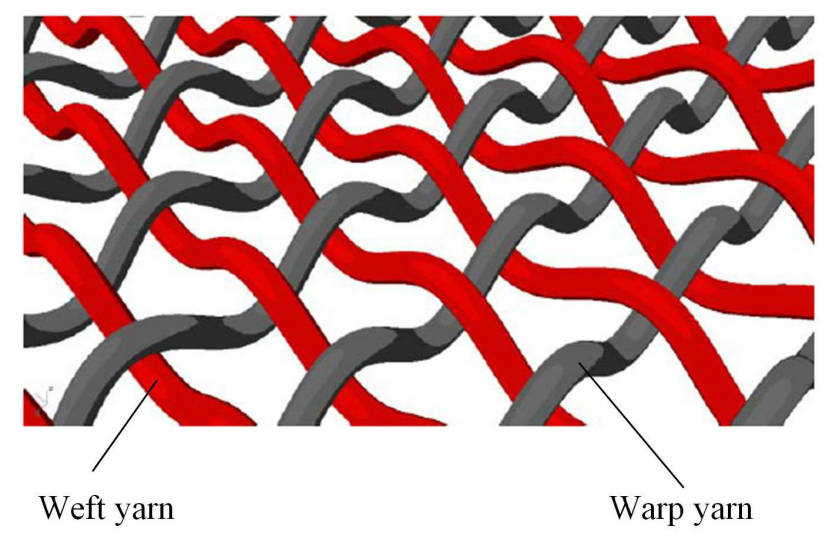

Figure 2. Complex fabric structure [9].

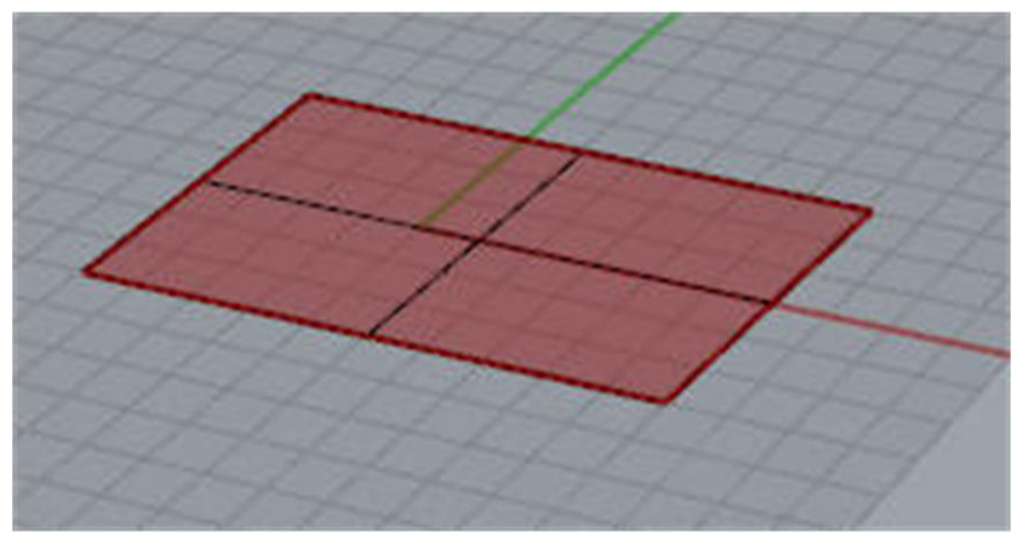

Figure 3. Determine the surface to be constructed. 


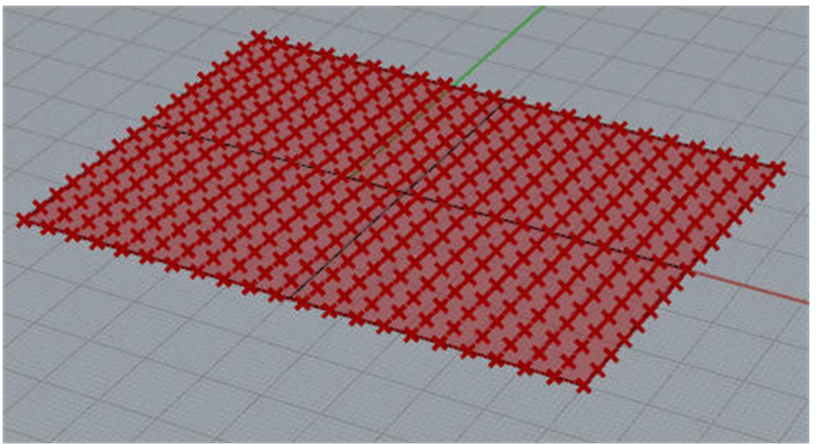

Figure 4. Dividing points on the surface.

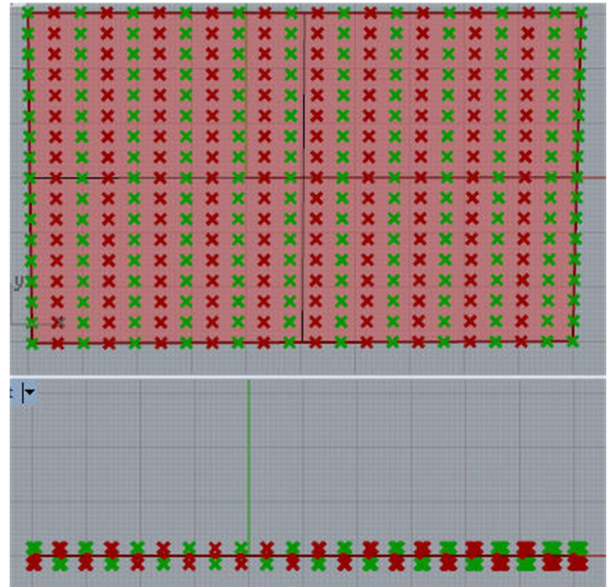

Figure 5. Weft positive and negative dividing points group.

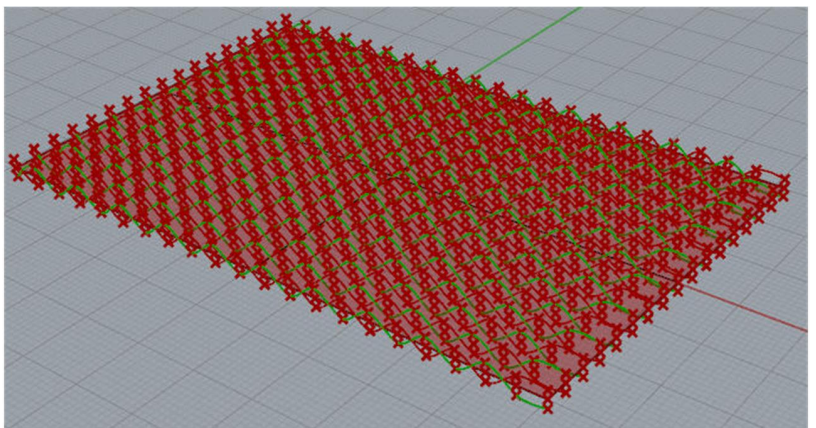

Figure 6. Weft trajectory curves.

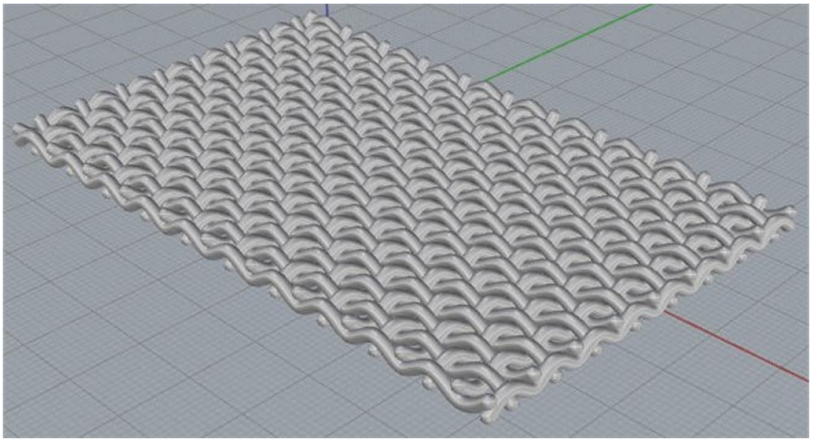

Figure 7. Finished pipe weaving characteristic. 


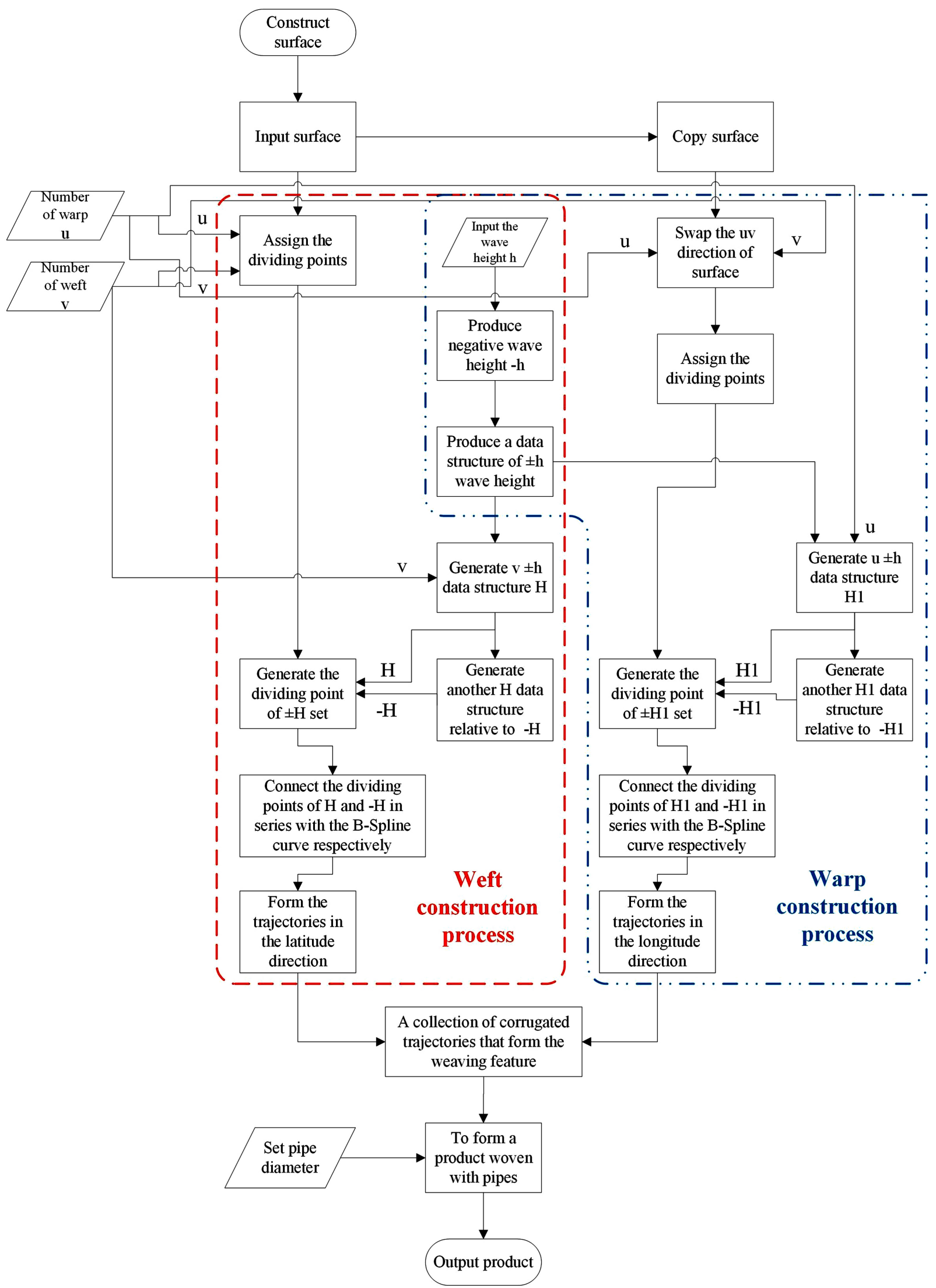

Figure 8. The flow chart of traditional construction process of products with weaving characteristics. 


\section{Parametric Design Principle}

Parametric design is a top-down calculation method, using related geometry as the basis, and then through the calculation of various dimensional parameters, as a technology of product design. Compared with traditional design methods based on subjective intuitive judgments, experience and habits, parametric design focuses on the definition of relationships. However, the setting process takes time to understand the logic behind and the choice of information. Zaha Hadid believes that the process of parameter importing design is necessary and can improve the feasibility of the design.

Parametric design uses the parameter rules of each characteristic as the design basis. Each parameter represents an important characteristic, which may be structure, capacity, cost or even some physical characteristics. By designing the interrelationship between the structure and data of each characteristic to generate the basic form required for the design.

In recent years, the application of parametric design techniques has gradually become popular. There are more and more CAD (Computer-Aided Design) software based on the concept of parametric and derivative design. However, most of the parametric derivative design tools are rarely packaged into a single standalone software at present, but mostly in the form of plug-in tools used on a specific CAD platform. For example, the parametric software "Grasshopper" developed by McNeel can be installed in the same company's Rhinoceros3D modeling software. It can be designed with the 3D model constructed by Rhino and can also be designed in Grasshopper. "Genoform" is based on Solidworks. Through Genoform, you can use the 3D model constructed by traditional modeling methods in Solidworks as the basis and parameterize the features of the model, so that you can quickly produce a large number of different types by adjusting each feature the design plan, the latest Genoform can also be linked to Grasshopper and Autodesk's Inventor software. In this study, the Grasshopper application plug-in to the Rhinoceros 3D modeling software was used as a modeling tool for weaving characteristic. The reason is that Rhino itself is easy to operate, and it has been widely used in the field of industrial design for modeling.

\subsection{Parametric Design Software-Grasshopper (GH)}

The Grasshopper is a graphical parametric design tool developed by the McNeel Corporation since 2007. It is installed in the 3D modeling software Rhinoceros produced by the same company in a plug-in form. Grasshopper is currently a software under work in progress, and many functions are often added and adjusted when the version number is updated. Grasshopper is mainly used to create generative algorithms. 3D geometric models are created through the connection, design of parameters and various calculators. The algorithms in the program are not only limited to the use of numbers, but text, video, audio, and touch applications can all be used as calculations or in the form of parameters. As architectural design is currently the most important field of parametric generative design 
methods, architects and related learners are currently the main users of Grasshopper, but its gradually improved and increasingly perfect graphical user interface and operation methods allow other fields users also began to pay attention to this software and used it as a tool for parametric design.

\subsection{The Modeling Concept of Grasshopper}

Grasshopper is a software that uses the graphics programming language (GPL) as its operating method. Simply put, GH uses Rhinoceros to create basic 2D and $3 \mathrm{D}$ geometric figures, edits the logic of the subsequent generation of geometric figures through $\mathrm{GH}$, and finally reuses Rhinoceros serves as its platform for presenting calculation results.

For example, in the past, to create a sphere in the Rhinoceros environment, it can be simply divided into several steps. First, draw the section contour line, use the rotation forming command, enter the rotation angle, and finally get the model. The entire construction process is shown in Figure 9(a). But when we need a semicircle, we have to operate the above process again. In the GH environment, we only need to find the most important arithmetic unit in the creation of a spherical surface: the rotating surface. When we understand the parameters required for the rotating surface, the axis of rotation, the section contour, and the angle of rotation, then through the interaction between the arithmetic units, it will automatically construct a complete sphere. The whole construction
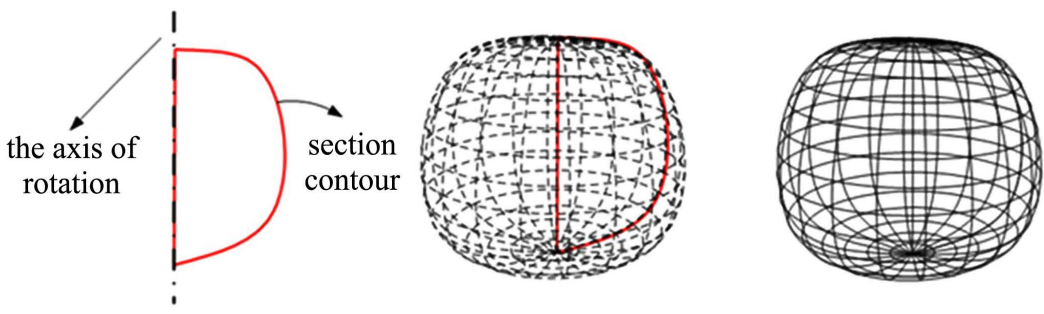

(a)

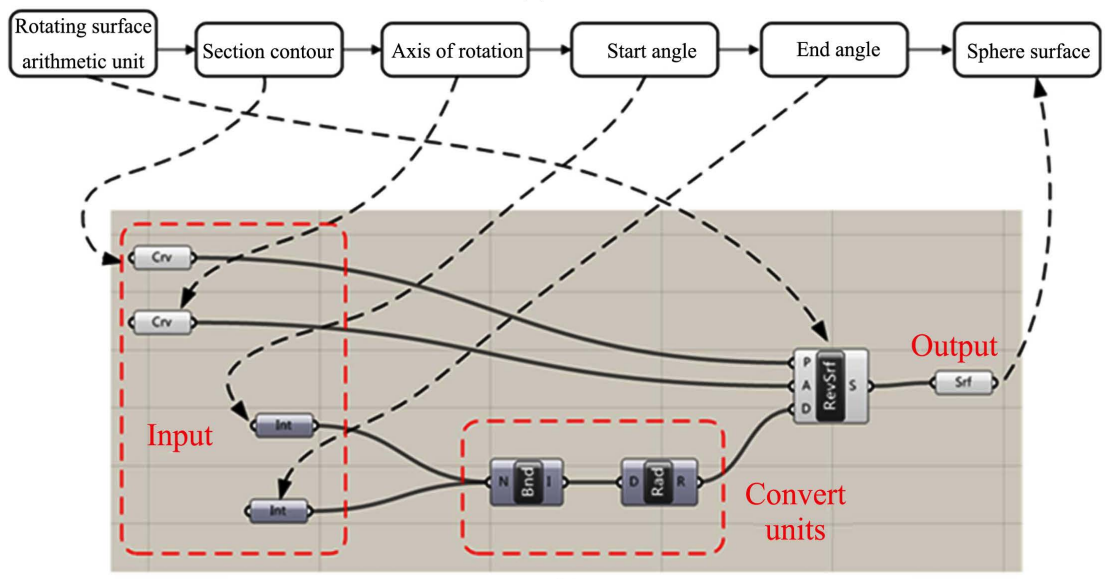

(b)

Figure 9. Differences and correspondence between Rhino and Grasshopper construction models. (a) Rhinoceros modeling method; (b) Grasshopper molding method. 
process is shown in Figure 9(b). Therefore, if we only need a semicircular surface, we can modify the rotation angle parameter immediately.

Taking the gradual change of the sphere as an example again, set the required number parts, and then use the arithmetic progression as the change of the radius parameter to complete the model as shown in Figure 10. When you want to change the requirements, you can quickly generate a new parameter model by adjusting the starting radius of the parameter and increasing the value.

\subsection{The Redesign Method of Grasshopper Operator Construction}

Parametric design and its open design operation platform makes the methods of constructing models very diverse. Designers can apply traditional modeling methods to construct various geometric modeling actions like piles of wood. Appropriate algorithms can also be added as an aid to produce various shapes. This derivative design method is the biggest feature and charm of parametric design.

This paper is to highlight the design change features of parametric modeling. Therefore, the size parameters that can be adjusted flexibly must be designed in the modeling process, and in the process of parameter adjustment, the appropriate relevance required by the modeling command must still be maintained. The following is an example of extruding 6 quadrangular pyramids from the 6 faces of a primitive cube in their respective normal directions to illustrate the GH operator construction flowchart, as shown in Figure 11. And Figure 12 is an example of the different results generated by inputting the extrusion height of 20 , 30 and $40 \mathrm{~mm}$ respectively [10].

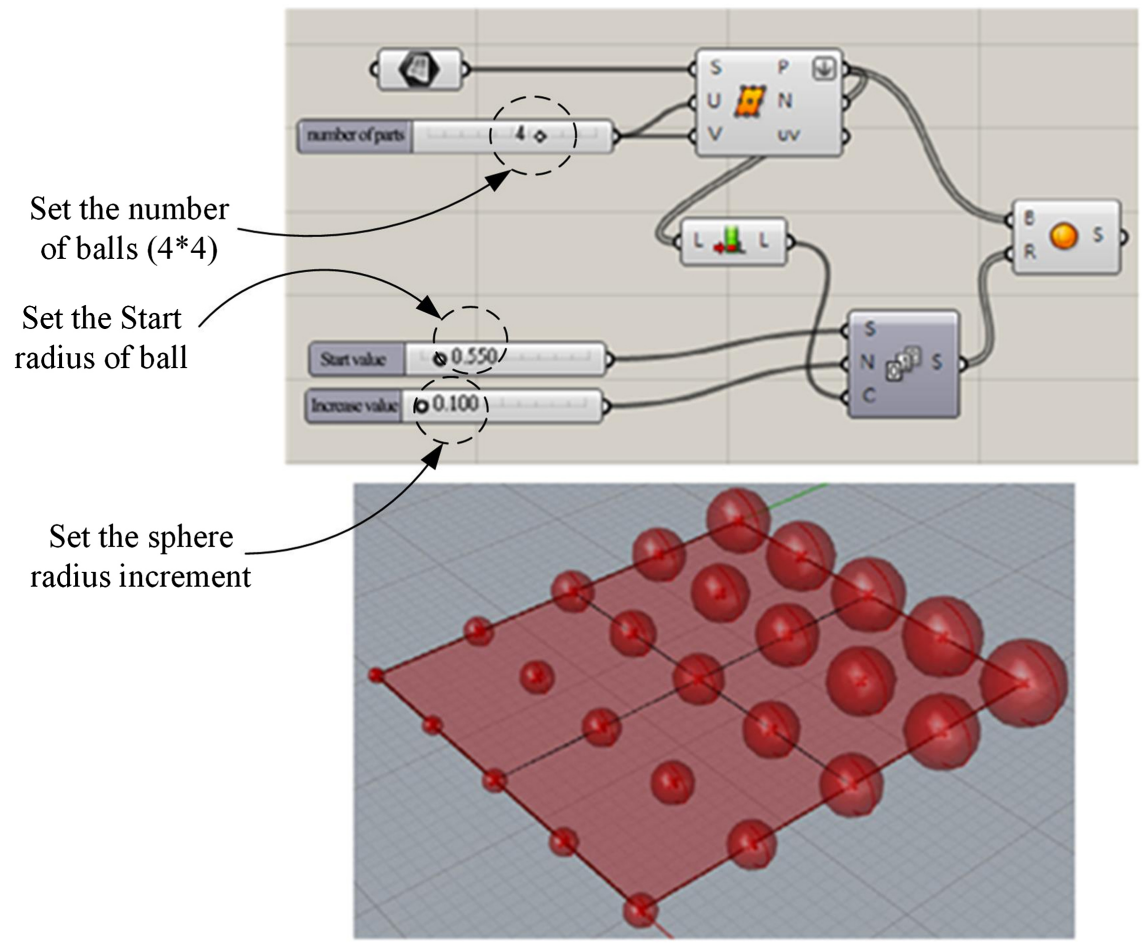

Figure 10. The Grasshopper's construction of gradient sphere model. 


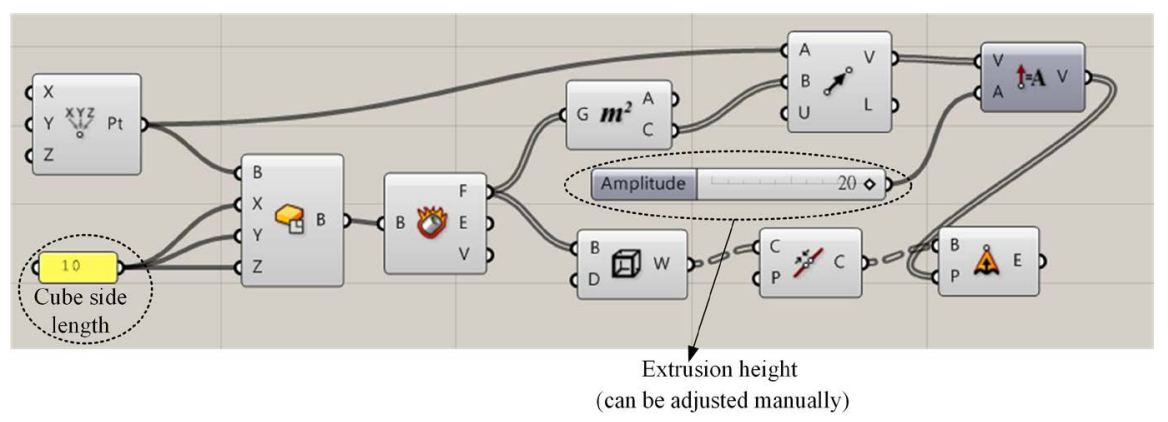

Figure 11. The Redesign method of GH operator construction flowchart.

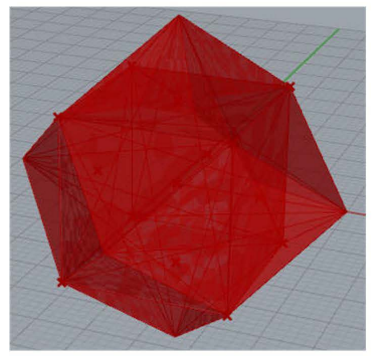

extrusion height 20

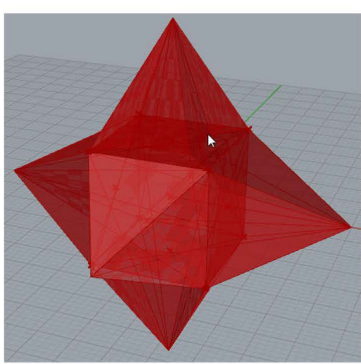

extrusion height 30

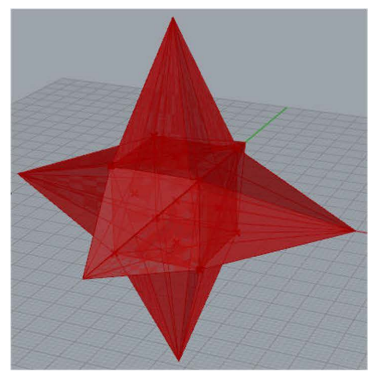

extrusion height 40

Figure 12. The different results generated by inputting the extrusion height of 20,30 and $40 \mathrm{~mm}$ respectively.

\section{GH Construction Process of Weaving Characteristics}

The calculation method of GH is mainly connected with each other through components, and generates results through layered parameter calculation and data analysis. Therefore, the steps of constructing waving characteristics through GH can be described as follows:

Step 1: Construct the surface to be waved in the Rhino environment and assign it to the GH surface component, as shown in Figure 13.

Step 2: Determine the number of warp and weft to get the number of dividing points, as shown in Figure 14.

Step 3: Determine the wave height. Used to control the offset value of the dividing points along their normal direction, as shown in Figure 15.

Step 4: According to the wave height and the number of warps and wefts, two data groups are generated: "Warp dividing points group" and "Weft dividing points group", as shown in Figure 16.

Step 5: Use the B-Spline curve to connect the dividing points of each warp and weft direction to generate multiple wave trajectories in series, as shown in Figure 17.

Step 6: Change all wave trajectories to round-head pipes with appropriate diameters to complete the finished pipe weaving characteristics, as shown in Figure 18.

Then if the $\mathrm{U}$ (Warp direction) and $\mathrm{V}$ (Weft direction) are reset to 20, 20, the result is shown in Figure 19. 


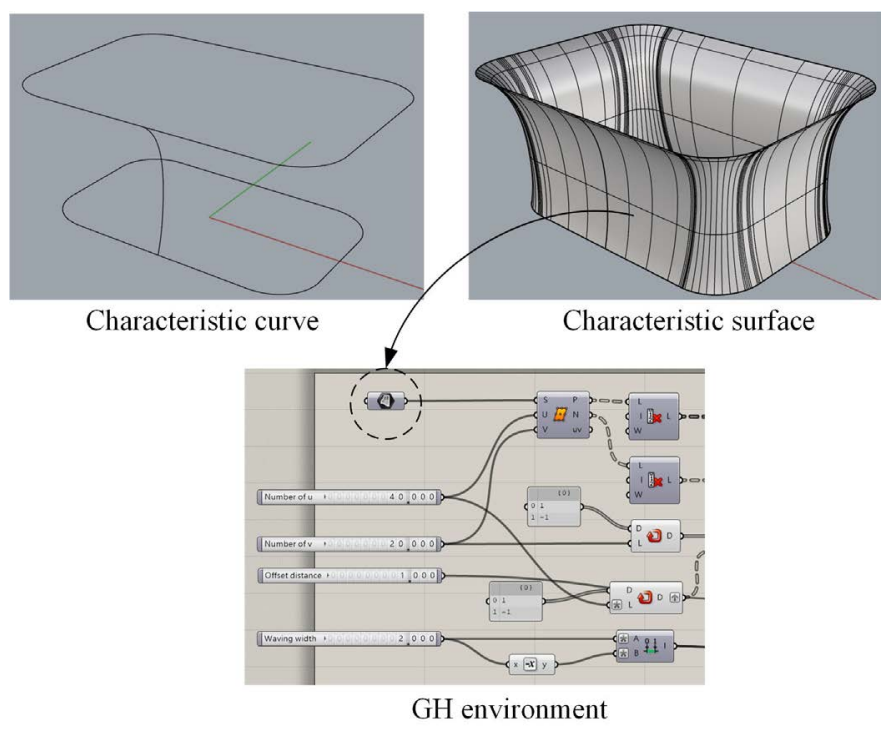

Figure 13. Link between Rhino object and GH component environment.

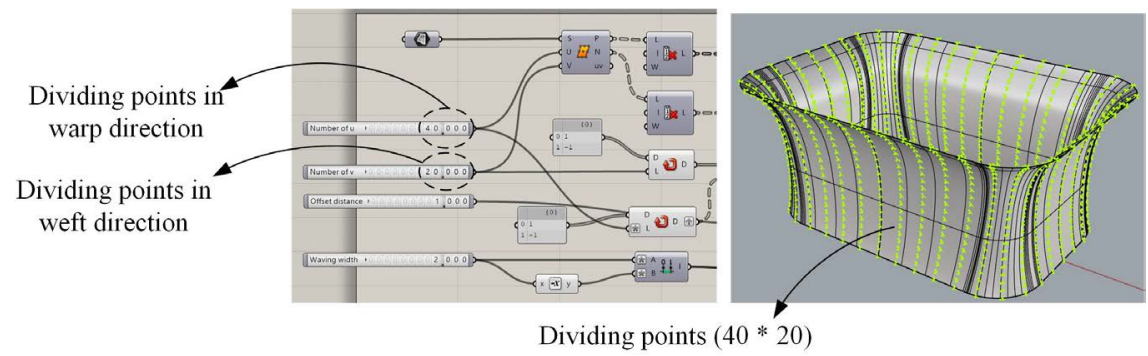

Figure 14. Dividing points on the surface.

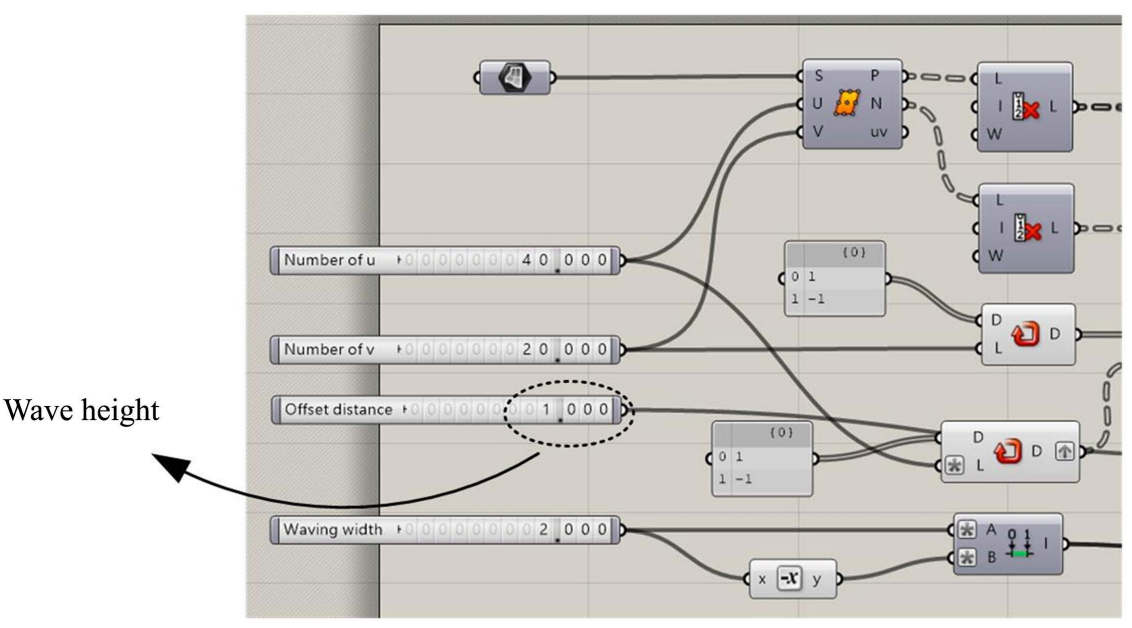

Figure 15. Set the wave height slider.

If $\mathrm{U}$ (Warp direction) and $\mathrm{V}$ (Weft direction) are reset to 20,10 , the result is shown in Figure 20.

In addition to changing the size of the pipe diameter, even changing the shape of the round tube can easily achieve the desired, as shown in Figure 21.

Figure 22 is the entire construction flow chart of GH module. 


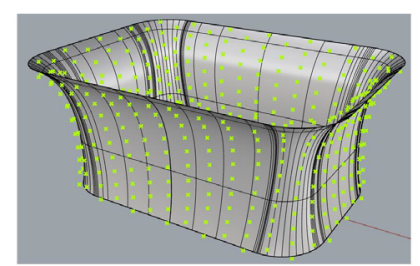

Warp dividing points

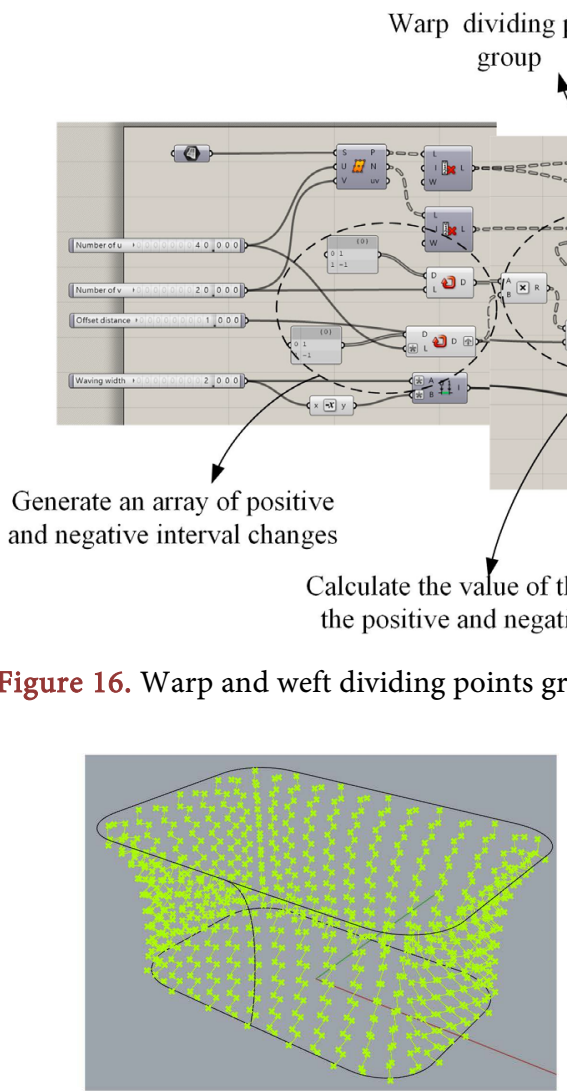

warp direction wave trajectories

Figure 16. Warp and weft dividing points group.

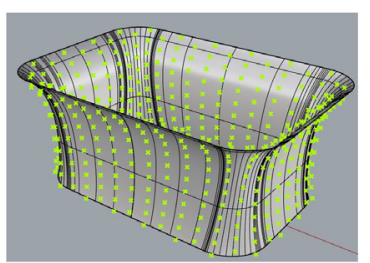

Weft dividing points group
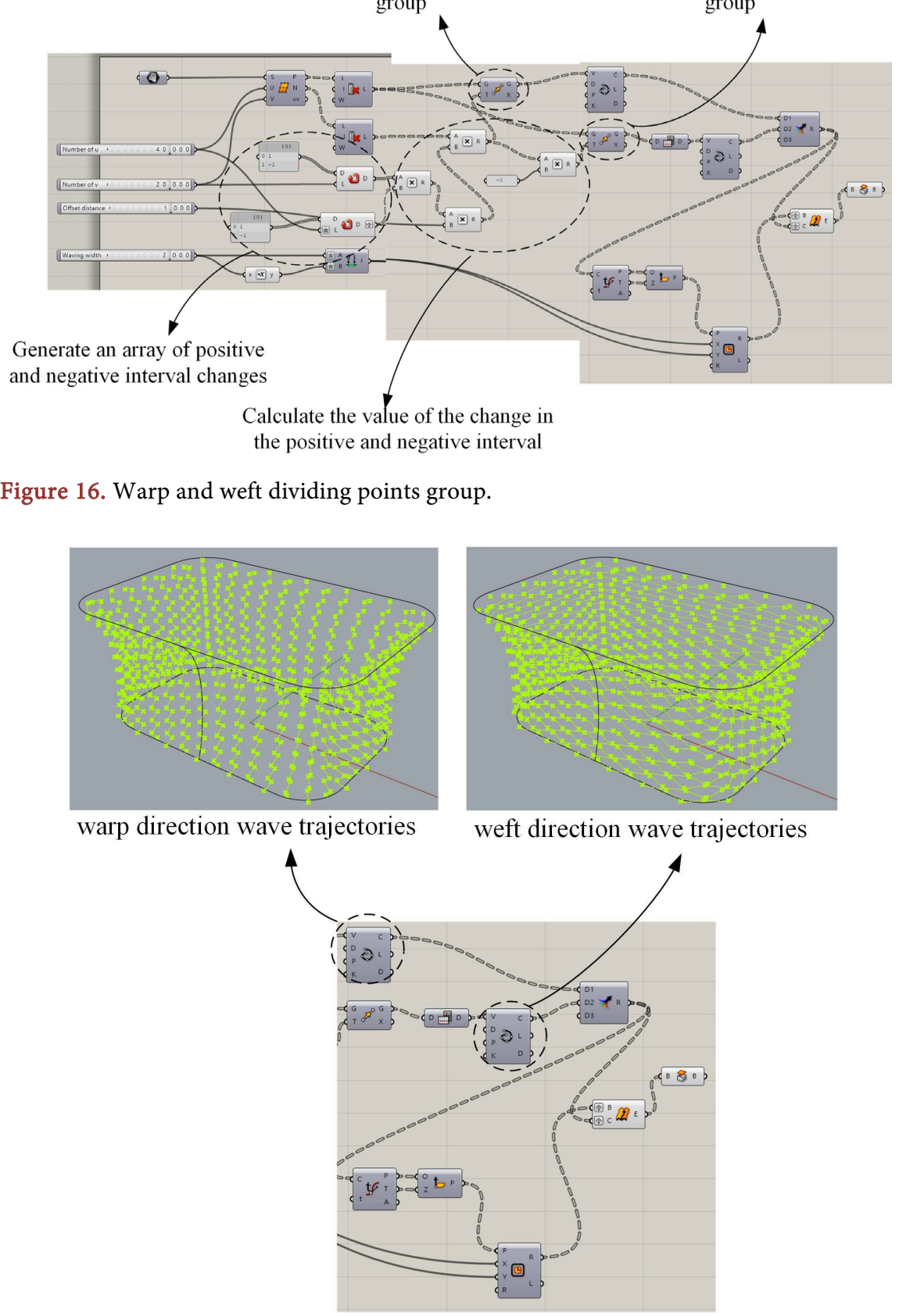

Figure 17. Warp and weft wave trajectories.

And Figure 23 is the finished models with weaving characteristics generated by different design parameters. Figure 24 is the finished products printed by the 3D printer. 

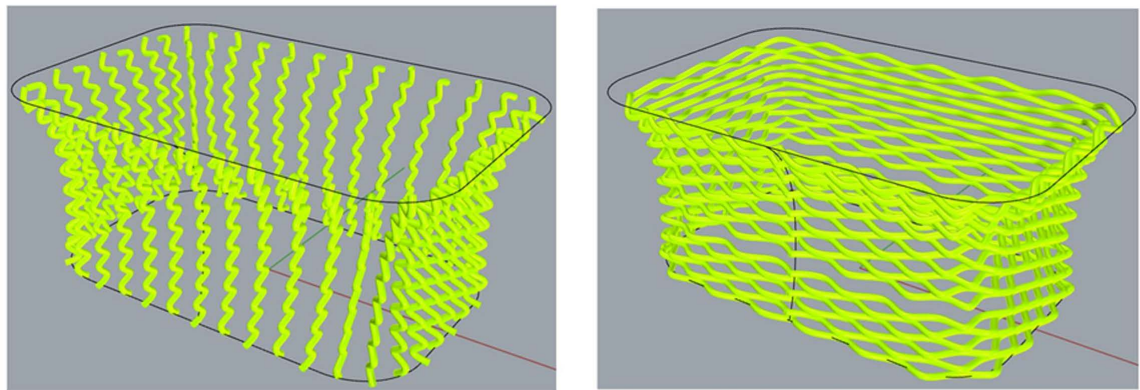

warp direction pipes

weft direction pipes

Combine

$+5$

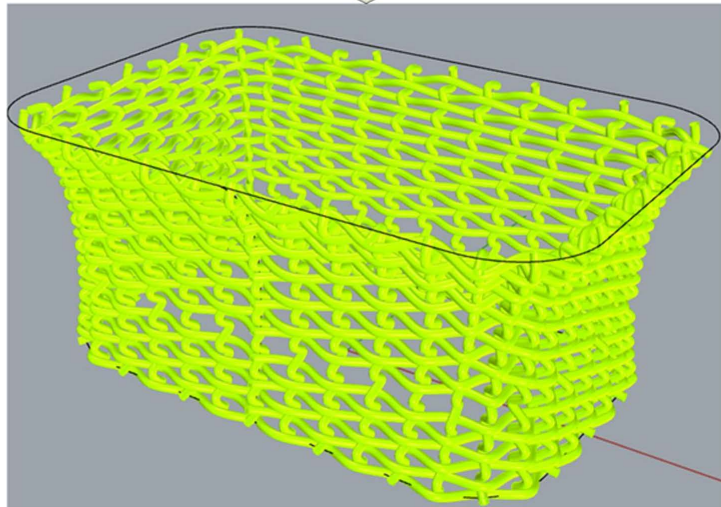

Figure 18. Warp and weft pipes.

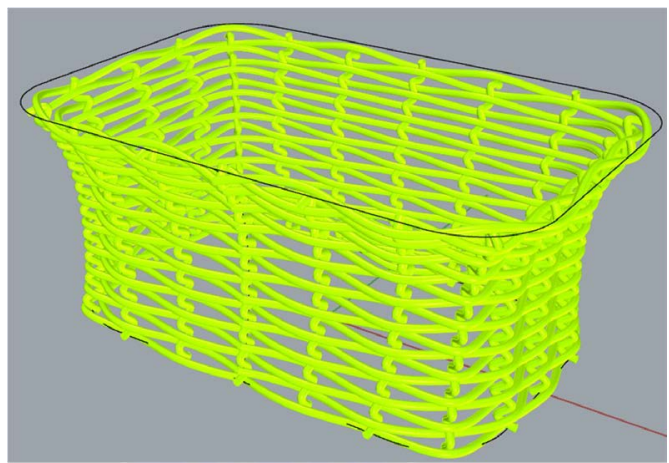

Figure 19. The result $U$ and $V$ are reset to 20, 20.

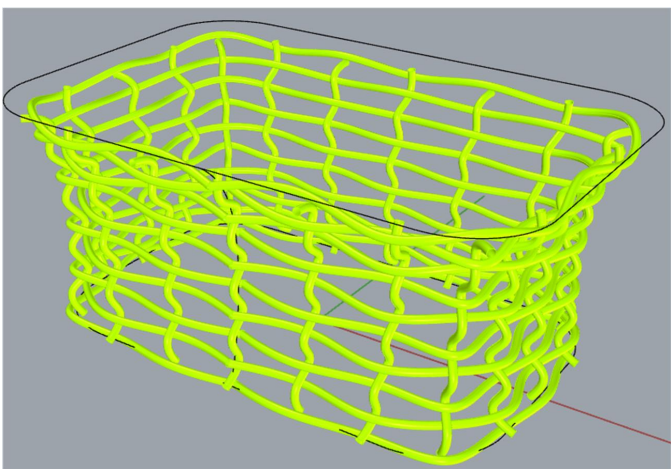

Figure 20. The result $U$ and $V$ are reset to 20, 10 . 


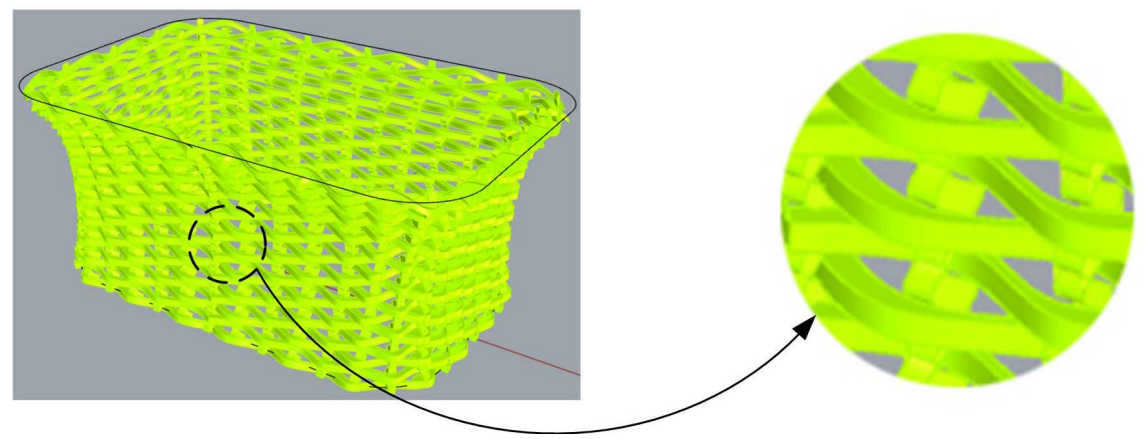

Figure 21. Flat shape of weaving feature.

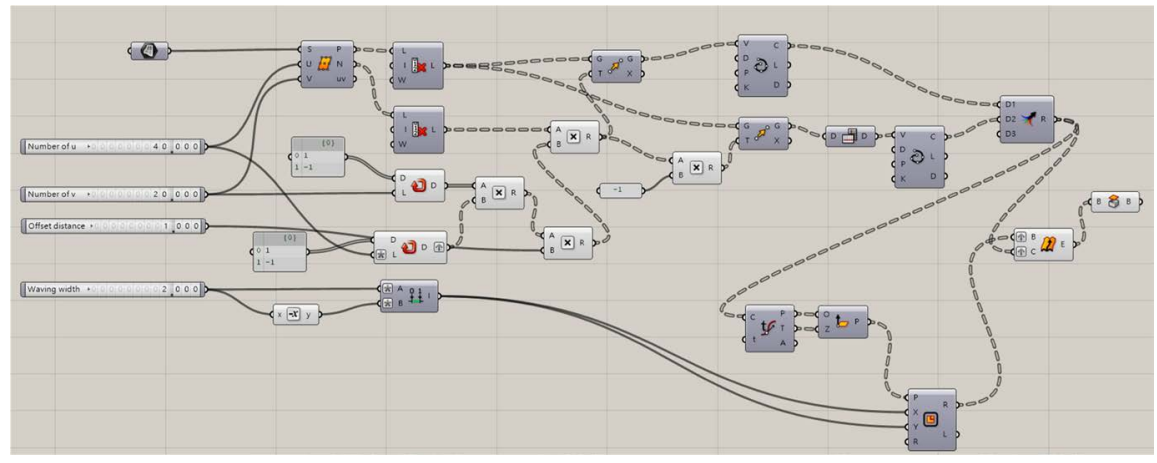

Figure 22. The entire construction flow chart of GH module.

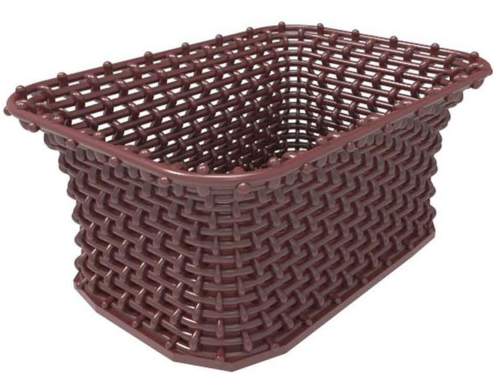

(a)

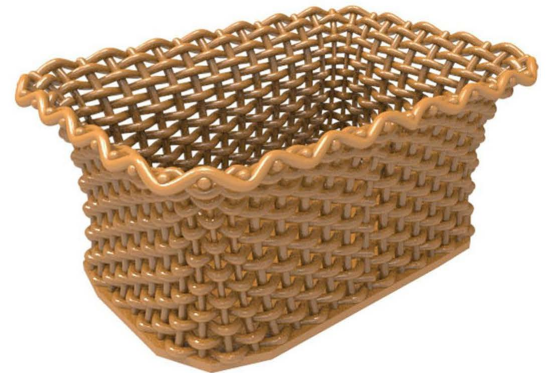

(b)

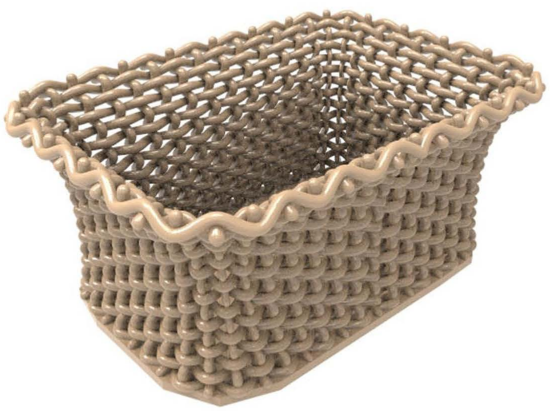

(c)

Figure 23. The models with weaving characteristics generated by different design parameters. (a) Model with warp weaving features; (b) Model with weft weaving features; (c) Model with weaving features in both warp and weft. 

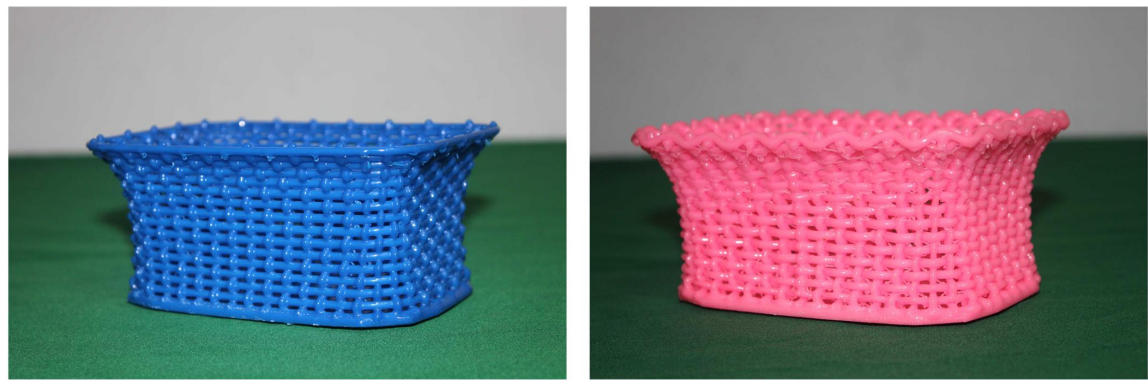

Figure 24. The finished products printed by the $3 \mathrm{D}$ printer.

\section{Conclusion}

This research uses parametric design software (Rhino + Grasshopper) as the tool to create products with waving characteristics, and verifies the actual application as the research objective. And by changing the number of dividing points of warp and weft, and the wave height, the design works can show the design change characteristics of parametric design. Without focusing on complex design theories, it only discusses how to effectively apply parametric design to the entire product creation process, and how to stimulate and guide designers to produce more possibilities. Under the constraints of the product itself, considering the compatibility between the existing digital processing methods and parametric design, this will explore the thinking and creative methods of parametric design, which can be used as a reference for subsequent learners. I hope that with the development of parameter design in the future, there will be more possibilities for application in the field of industrial design.

\section{Conflicts of Interest}

The author declares no conflicts of interest regarding the publication of this paper.

\section{References}

[1] Hua, H.J. (2016) The Creation of Weaving Image Pingtung-The Modern Application of Weaving Techniques. Master's Thesis, National Pingtung University, Pingtung.

[2] Chen, Y.Z. (2005) A Romantic Dialogue between Weaving and Art-A Creative Study of the Combination of Weaving Craftsmanship and Modern Ink and Wash. Master's Thesis, Taipei Normal University, Taipei.

[3] Krish, S. (2011) A Practical Generative Design Method. Computer-Aided Design, 43, 88-100. https://doi.org/10.1016/j.cad.2010.09.009

[4] Chen, Z.C. (2011) Digital Program of Architectural Design. Architect, 66-69.

[5] Huang, S.W. (2011) The Cradle Architect of World-Class Architecture. Zaha Hadid Architects, 37, 124-129.

[6] You, S.B. (2012) Natural Mode: The Application of Parametric Design in Product Shape Creation. Master's Thesis, National Chiao Tung University, Hsinchu.

[7] Li, S.Y. (2013) Parametric Design Is Used in Product Creation and Discussion. Master's Thesis, National Chiao Tung University, Hsinchu. 
[8] Zhang, Z.J. (2011) Empirical Case Study of Parameter Design and Digital Manufacturing. Master's Thesis, National Taiwan University of Science and Technology, Taipei.

[9] Khabazi, Z. (2010) Generative Algorithms Concepts and Experiments: Weaving. http://www.morphogenesism.com

[10] Chang, H.-C. (2021) Parametric Design Techniques Applied to Creative Hollow out Product Design with 3D Voronoi Patterns. Journal of Computer and Communications, 9, 32-47. https://doi.org/10.4236/jcc.2021.98003 